\title{
Dire et écrire l'expérience pour réguler son agir professionnel : réflexions à propos d'un « genre réflexif académique »
}

Sabine Vanhulle

\section{(2) OpenEdition \\ Journals}

Édition électronique

URL : http://journals.openedition.org/pratiques/3205

DOI : 10.4000/pratiques.3205

ISSN : 2425-2042

Éditeur

Centre de recherche sur les médiations (CREM)

Référence électronique

Sabine Vanhulle, « Dire et écrire l'expérience pour réguler son agir professionnel : réflexions à propos d'un « genre réflexif académique » », Pratiques [En ligne], 171-172 | 2016, mis en ligne le 07 février 2017, consulté le 10 décembre 2020. URL : http://journals.openedition.org/pratiques/3205 ; DOI : https://doi.org/10.4000/pratiques.3205

Ce document a été généré automatiquement le 10 décembre 2020.

(c) Tous droits réservés 


\title{
Dire et écrire l'expérience pour réguler son agir professionnel : réflexions à propos d'un " genre réflexif académique »
}

\author{
Sabine Vanhulle
}

\section{Introduction}

1 Exercice étrange que la réflexivité discursive: elle interpelle la personne dans la mise à plat d'actes et de gestes réalisés ou projetés, de doutes et de questions sur ses expériences pratiques; et elle l'incite en même temps à mobiliser des capacités d'analyse, de conceptualisation et de mise en textes pour en dégager des savoirs professionnels. Cette démarche réflexive constitue ainsi la clé de voûte entre, d'une part, un réel vécu subjectivement qu'un langage reconfigure, et, d'autre part, l'intégration de ressources théoriques et scientifiques dans un discours académiquement pertinent.

2 Mais quel rapport entre réflexivité et écriture ? Ni descriptive, ni expressive, ni narrative, ni argumentaire, mais tout cela à la fois, cette écriture-là a une fonction avant tout structurante, engageant un processus d'enquête (Dewey, 1993, 2011). L'enseignant en formation est appelé à identifier des situations de travail qui l'incitent à décider de ses actions et à en jauger les conséquences. Ces situations contiennent des parts d'indétermination, d'opacité. Il lui faut trouver les mots pour saisir en quoi ces situations - éprouvées, subies ou agies - constituent pour lui une expérience d'apprentissage. Expérience parfois cruciale si elle enclenche la restructuration de concepts dans l'interprétation de l'activité professionnelle, débouchant sur une généralisation objectivante au-delà des situations locales (Vygotski, 1997).

3 Nous envisageons ici l'usage académique de l'écriture réflexive sous l'angle d'un genre réflexif académique". Se déployant dans un continuum de divers types de textes oraux et 
écrits, ce genre vise le développement des enseignants selon trois grands axes impliquant la réflexivité : s'approprier des savoirs de référence pour la formation (Hofstetter \& Schneuwly, 2007 ; Marcel, 2010) ; intégrer une identité professionnelle ; réguler concrètement son agir.

\section{Le genre réflexif comme lecture régulatrice de l'agir}

\section{Une perspective interactionniste sociodiscursive}

4 Nombreux sont les dispositifs de formation initiale des enseignants qui invitent à l'écriture (Dufays \& Thyrion, 2004) pour s'approprier le métier, prendre conscience des compétences et des savoirs à acquérir et mobiliser, construire un éthos discursif (Delormas, 2012 ; Dobrowolska, 2014) et professionnel (Jorro, 2011 ; Mosquera, 2014).

Le genre réflexif aurait aussi pour fonction de susciter des régulations (Buysse, 2009) par la personne de son agir professionnel, en se penchant sur l'intelligibilité des situations de travail (Schön, 1996 ; Perrenoud, 2001). Ce qu'il vise donc, ce sont des transformations - y compris de soi-même en tant que professionnel - impulsées par l'analyse approfondie de l'expérience effective de ces situations (Dewey, op.cit. ; Mezirow, 2001). Cette optique est évidemment pragmatique. C'est un aspect du genre.

Dans une perspective interactionniste sociodiscursive (Bronckart, 1996) imprégnée notamment des thèses de Vygotski $(1997,2003)$, ce genre peut remplir aussi une fonction épistémique importante, si l'on admet comme nous le faisons les présupposés théoriques qui suivent. Le langage verbal, au-delà de ses fonctions expressive et communicative, soutient le développement de la conscience par l'appropriation des outils d'intervention dans le monde qui se sont forgés dans la culture. Parmi ces outils figurent les concepts véhiculés dans les mots. Par le retour de la pensée sur elle-même qu'ils permettent, les mots, chargés de concepts, sont des instruments de construction de sens au-delà de l'usage pertinent de significations consignées dans les dictionnaires. Médiatisant la formation de concepts autant que le rapport de la personne à une réalité façonnée par la culture, le langage inscrit la personne et son agir dans une historicité.

7 En suivant Bronckart (2005), les régulations de l'agir enclenchées dans le processus réflexif qui est par définition langagier renvoient, au-delà des réajustements que le sujet opère au niveau de ses actions concrètes, à sa lecture de ce que signifie et implique sa participation à l'activité professionnelle ${ }^{1}$.

\section{Quelle place pour les écrits professionnels dans le genre réflexif ?}

8 L'écriture professionnalisante, en formation initiale, ne se superpose pas nécessairement à l'appropriation des écrits spécifiques de la profession enseignante (Daunay, 2011). En analysant les pratiques des enseignants autour de la constitution de supports pour leurs propres tâches (par exemple, la planification des leçons) et pour guider l'activité des élèves, Morisse (2012) constate que ces écrits ne sont pas identifiés par leurs auteurs comme des outils réflexifs : "la fonction heuristique de l'écriture est sous-estimée, alors qu'elle est susceptible de contribuer à l'analyse de la pratique de l'enseignement, notamment en cas d'échec" (p. 172). 
9 L'écriture n'est que rarement investie comme un outil de réflexivité dans les pratiques enseignantes, malgré la masse d'écrits professionnels que les enseignants sont amenés à rédiger - de la préparation de leçons aux constructions collectives de documents tels que supports pédagogiques et didactiques partageables sur internet ou projets d'écoles soumis aux autorités, en passant par les sanctions écrites ou encore les commentaires évaluatifs dans les bilans et portfolios d'élèves. Comme le suggère Morisse (2012), ces textes professionnels s'intégrant dans l'activité de travail, ils mobilisent avant tout une forme de réflexivité plus tacite et plus immédiate ; en reprenant les termes de Giddens (2005), ils relèvent d'une conscience pratique et non discursive.

On peut en conclure sans trop de risques que le genre réflexif en tant que tel relève d'une invention académique, visant un idéal de réflexivité critique au-delà des préoccupations en termes de "comment faire". Dans ce sens, puisque le genre réflexif s'appuie en particulier sur des écrits d'apprentissage qui seraient censés amener les étudiants aux écrits professionnels (Beaudet \& Rey, 2012), cela implique la lecture - au sens de Bronckart (supra) - des contraintes de l'agir professionnel et des régulations qu'il suppose dans l'urgence et l'incertitude, montrant ainsi aux étudiants que la pratique réflexive comme attente académique (Leblay, 2014) tend à les relier concrètement à leur profession précisément par la fonction de distanciation qu'elle suggère.

\section{Un processus de subjectivation}

11 Parce qu'elle est réflexive, la mise en discours de leurs apprentissages par les enseignants en formation procède à des degrés variables d'une subjectivation (Vanhulle, 2009, 2011) : traduction des significations offertes dans un discours singulier en quête de cohérence, mise en scène d'un soi professionnel qui s'approprie des marges de manœuvre tout en intégrant les savoirs, normes ou prescriptions en vigueur.

Cette mise en discours du soi et de ses savoirs se déploie dans un continuum permettant toutes sortes d'interactions de l'oralité à l'écriture et vice versa. Du côté de l'écrit: protocoles d'analyses de pratiques basés sur des référentiels de compétences ou de gestes professionnels ou sur des outils pédagogiques ou didactiques d'observation et d'analyse, journaux de bords suscitant peu ou prou la mise en scène de soi et de son vécu, portfolios et autres écrits de formation (Ricard-Fersing \& Crinon, 2004) impliquant la manipulation de connaissances théoriques, le rapport de stage, l'analyse d'incidents, l'analyse des leçons réalisées ou des planifications de séquences et de leurs réajustements en cours d'action, les récits d'événements, les fiches de résumés d'ouvrages de référence, les travaux d'élèves annotés, jusqu'aux récits de longue tenue (Pellanda-Dieci \& Tosi, 2014 ; Pellanda-Dieci \& al., 2012 ; Vanhulle, 2009). Du côté de l'oral: participations à des interactions sur les mêmes types d'objets, entretiens de stages notamment structurés par des référentiels, guides et consignes académiques organisant les modalités d'échanges entre les enseignants en formation (désormais $\mathrm{EF}$ ), tuteurs de terrain $(\mathrm{T})$ et superviseurs de l'institut de formation (S), entre la conversation et l'entretien de type explicitation ( Vermersch, 1994) et autres entretiens dyadiques ou tripartites d'analyse des pratiques ou d'évaluation de stages (Balslev \& al., 2014; Balslev \& Tominska, 2012).

Ces différentes pratiques discursives entrainent la production de textes, au sens où ces discours impliquent de délivrer un contenu organisé par la recherche d'une cohérence (Bronckart, 1996: 137). De l'oral structuré par des échanges avec des pairs et des formateurs à l'écrit le plus élaboré, ce contenu renvoie à la formulation de 
problématiques professionnelles à partir de "situations questionnantes" (Pellanda-Dieci et al., op.cit.) ; à des analyses épistémiques de concepts scientifiques et de leurs implications en termes de réflexion pour la pratique ; à des délibérations sur des normes d'organisation du travail scolaire ou sur des questions éthiques ; à la mise en perspective de phénomènes éducatifs à partir de narrations ${ }^{2}$.

\section{Des exigences littéraciques de haut niveau}

14 Avec les rapports de stages ou les protocoles d'analyses didactiques de leçons ou de travaux d'élèves, entre autres, qui élargissent déjà l'éventail des textes académiques habituels, la production de textes réflexifs écrits et oraux vient s'ajouter aux exigences en matière de littéracie universitaire (Blaser \& Pollet, 2010 ; Delcambre \& Lahanier-Reuter, 2012) et d'écriture professionnelle (Morisse, 2012). Les nouvelles compétences langagières attendues à l'université en lien avec un but de professionnalisation, auquel la réflexivité renvoie, sont encore largement à cerner, d'autant plus que ces textes sont le plus souvent sollicités dans les institutions de formation de manière éparse selon les cours et les domaines disciplinaires et selon des objectifs eux aussi multiples.

Posons au moins ceci: la réflexivité engage le sujet dans des opérations mentales singulièrement complexes. Entre autres, les énoncés attendus ont à se situer entre implication et distance, sous des aspects de "réfléchissement de l'action dans le dire" alors que le langage n'est pas une traduction de la réalité mais une interprétation; de "réflexion sur l'action" menée alors que l'énonciateur n'a pas d'accès direct à sa propre expérience ; et de "métaréflexion sur le dire à propos de l'action" (Morisse, 2003) alors que l'action n'est pas encore identifiée par les débutants en fonction d'une connaissance approfondie de la profession et des actions, compétences, savoirs et attitudes qu'elle implique.

En outre, les EF ont à s'intégrer dans un ensemble de significations proposées par les formateurs-chercheurs, fixées dans les référentiels de la formation, en partie orientées par des exigences politico-administratives en termes de compétences attendues et de curriculums scolaires. Les injonctions externes comme les offres de savoirs de référence sélectionnés dans les dispositifs académiques, conduisent nécessairement les futurs enseignants à s'inscrire - au sens le plus strict du mot puisqu'on les fait écrire à ce sujet dans une formation discursive marquée par des savoirs provenant de la recherche mais en même temps transformés à l'extérieur dans des doxas institutionnelles et dans des présupposés de sens commun. Ces différentes bases référentielles «scripturalisent " littéralement les cadres de l'activité enseignante.

\section{2. À la recherche des traits du genre}

17 Dans cet horizon de pensée et de discours, ainsi que Volochinov $(1981,2010)$ l'a thématisé il y a un siècle, la mise en œuvre des formes linguistiques par le locuteur ne constitue pas une expression de ses propres orientations psychologiques ; elle sollicite des formes déjà chargées de valeurs sociales qui reflètent les tendances qui sont dominantes ou stabilisées dans un contexte social déterminé (Bronckart \& Bota, 2011 : 446). 


\section{Tension entre la situation d'énonciation et la mise en scène des situations vécues}

est donc dans ce contexte balisé que la prise en charge des contenus référentiels doit s'associer à des formes d'énonciation subjective liée à l'expérience singulière de formation. Celles-ci n'interdisent pas - et pour cause - l'effacement énonciatif, en particulier lorsque les thèmes abordés quittent la narration de l'expérience au profit de propositions théoriques : comme le soulève Rabatel (2005), l'énonciation théorique s'accommode "de la très forte présence de subjectivèmes exprimant un point de vue, alors que le locuteur a choisi de présenter ces objets du discours dans des énoncés non embrayés ; l'absence de déictiques n'implique pas l'absence de marques modales, bien au contraire (...)" (Rabatel, 2005, p. 117).

ops illustre parfaitement l'un des traits observables du genre réflexif, dès lors que l'EF doit endosser une posture d'énonciateur quant à des références scientifiques qui font autorité, que modestement il ne s'autorise pas à s'approprier dans un "je" généralement proscrit dans le contexte académique. Tension, donc, puisqu'il lui est en même temps demandé d'exprimer dans ce contexte-là d'énonciation un point de vue de professionnel à propos d'une situation de travail vécue hors de ces murs et dont il est un témoin et un acteur.

Ce changement de posture induit une prise de risque, voire une insécurité discursive face à la gestion des jargons théoriques de la recherche en éducation (Moirand, 2000 ; Vanhulle, 2015). Dans ce cas, l'évitement de contenus théoriques peut le disputer à l'effacement énonciatif derrière un discours purement restitutif, non embrayé mais aussi peu modalisé.

21

Retenons donc que le genre réflexif provoque une tension entre la situation énonciative interne à l'université et la mise en scène de la situation vécue : la "scène énonciative" (Maingueneau, 2013) n'est pas la scène de la réalité. Tout se joue alors dans la manière dont le locuteur/ énonciateur (Rabatel, 2009) se met en scène dans la représentation (Grize, 1996) de l'expérience que son discours donne à voir, à travers la scénographie que constitue l'éthos discursif (Maingueneau, ib. ; Dobrowolska, 2014) et ses ruses.

\section{Expérience et energeia}

Que signifie alors l'analyse réflexive des "situations expérienciées" (Dewey, 1993) ? Enquête, certes, portant sur la valuation (Dewey, id.) des rapports entre les actions et leurs conséquences, et débouchant sur la production de valeurs (id.). Mais l'injonction académique prend au sérieux la dimension "scientifique" qui rapproche la démarche réflexive d'une démarche de recherche. C'est donc aussi à une quête décontextualisée de concepts qu'il faut s'adonner, s'ouvrant à des sources possibles de généralisation au sens de Vygotski :

Le mot absorbe les contenus intellectuels et affectifs tirés du contexte dans lequel il est inséré, il s'en nourrit et se met à signifier plus ou moins que ce qui est contenu dans sa signification lorsque nous le considérons isolément, en dehors du contexte : plus parce que le cercle de ses significations s'élargit, acquérant encore toute une série de zones chargées d'un nouveau contenu ; moins, parce que la signification abstraite du mot est rétrécie et limitée à ce que le mot signifie dans le seul contexte donné » (Vygotski, 1997 : 481). 
Cette mise en recherche n'est pas purement hypothético-déductive, mais compréhensive. La mise en discours réflexive de l'expérience relève dès lors d'une véritable création de sens, prenant vie dans les ressources créatives de la langue comme energeia (Coseriu, 1968).

Dire ou écrire le vécu en lui donnant sens, c'est à proprement parler une production dynamique de signes, dans leur double facette de contenu et d'expression. Le genre réflexif invite à inventer des formes d'appréhension de la réalité expérienciée. Mais il s'agit encore que ces formes puissent être reconnues et élaborées au plan intersubjectif: pour Coseriu, c'est la base même sur laquelle l'expérience humaine peut s'organiser (Bota, 2008). C'est à cela que peuvent servir par exemple les entretiens et les écrits réflexifs : à proposer des appréciations partageables des situations étant donné les conditions sociales qui orientent les échanges et débats (y compris dans la gestion des textes écrits) : "l'orientation sociale est précisément l'une de ces forces vivantes et constitutives qui, en même temps qu'elles organisent le contexte de l'énoncé - la situation - déterminent aussi sa forme stylistique et sa structure grammaticale" (Volochinov, 1981, cité par Bronckart \& Bota, 2011 : 457).

Voici deux exemples de ces écrits sollicités dans les dispositifs que nous analysons :

- Textes présentant une "problématique professionnelle" exposée aux formateurs dans les entretiens oraux sur la base d'un texte préalablement rédigé comme support structurant l'exposé (dans la formation en enseignement primaire) ${ }^{3}$.

- Micro-récits de situations dites "questionnantes" (dans la formation en enseignement secondaire $)^{4}$.

L'analyse des premiers par Dobrowolska (2014) a permis de mettre en évidence un rapport étroit entre la structuration apportée par l'EF à son support de présentation, et le degré de présence de savoirs professionnels. Ceux-ci sont plus clairement formulés lorsque le texte écrit se divise de manière systématique en trois parties : description d'une situation concrète dans laquelle la problématique prend son ancrage ; analyse des composantes de cette situation; généralisation en termes de problématique récurrente de l'activité enseignante (Dobrowolska, 2014).

Quant aux micro-récits de situations questionnantes, ils s'appuient sur des événements plus ou moins critiques à partir desquels les EF élaborent des questions professionnelles auxquelles ils tentent d'apporter des réponses généralisables en s'appuyant sur les régulations qu'ils ont pu mettre en place et en les étoffant théoriquement (Pellanda-Dieci \& Tosi, 2014). Un constat majeur ressort de l'analyse de ces micro-récits qui entrent dans les DDP et sont repris, étendus, enrichis au fil du parcours de formation pour s'intégrer au final dans une réflexion plus généralisante à travers un récit final de formation.

Ce constat est que le degré de construction des savoirs professionnels est à la mesure des préoccupations exprimées par les EF dans leurs récits (et dans leurs écrits réflexifs en général, dans les deux formations), avec tous les sous-jacents - ensembles de croyances et d'affects - qui orientent la conduite de leurs actions - rapports aux élèves et aux savoirs scolaires, sentiments propres d'efficacité, estime de soi, autoévaluation en termes d'autorité et de gestion de la classe, etc. 
30 Dit autrement, le genre réflexif peut encourager l'expression de ces préoccupations et affects. Par là, pour autant que la personne en formation s'engage dans les formes de discours qu'il sollicite entre implication et distance, il permet de relier la pensée verbale et le corps, et potentiellement de mettre en lumière des savoirs incarnés dans la conscience pratique.

31 La constitution du soi professionnel en découle, dans la mesure où de tels savoirs sont authentiquement le produit des expériences, dans la mesure où celles-ci sont sémiotisées, couchées sur le papier ou échangées avec autrui. Cela semble se confirmer au-delà de la formation initiale (Mosquera, 2014): selon ce chercheur, la subjectivation de savoirs professionnels à partir de préoccupations vécues en stages de formation initiale soustend la pratique de l'enseignant en début de carrière.

\section{Une activité langagière incarnée}

32 À une focalisation sur les "situations expérienciées" chères à Dewey, qui sans langage seraient inatteignables en tant que telles et condamnées à l'indétermination, doit ainsi clairement se substituer, pensons-nous, l'idée de la situation travaillée comme une sémiotisation de l'agir, "redoublement de l'expérience" (Vygotski, 2003) à travers l'activité langagière. Nous soutenons que c'est dans le processus même de cette sémiotisation que, comme l'affirme Bulea (2011), les compétences langagières s'articulent aux compétences professionnelles.

33 Tout genre de discours se définit par ce "caractère biface" (Branca-Rosoff, 1999 : 116), qui fait correspondre une face interne (les fonctionnements linguistiques de la mise en discours) avec une face externe (les pratiques sociales d'appartenance). En l'occurrence, comme on l'a vu, le genre réflexif est marqué par son inscription dans le milieu académique ; mais pour les EF il s'imprègne aussi fortement du contact avec le milieu professionnel et de leur expérience soutenue de l'activité d'enseigner jour après jour durant de longues périodes.

34 Il y a donc, dans ce genre, tension entre acculturation de l'étudiant au monde académique, et acculturation du stagiaire au monde professionnel. Cette tension est heuristique en ce qu'elle offre des opportunités d'articuler les registres discursifs académiques et professionnels. Partant de la relecture des concepts - ainsi que des normes et prescriptions - issus de la recherche, associé à la relecture des situations expérienciées, le genre réflexif soutient un travail de traduction de la recherche en pratiques, procédant par tâtonnements, par "une ingéniosité rusée qui se développe dans la nécessité du moment présent et qui, sans être entièrement prise en charge par la raison (...) demeure source effective de savoir. Ce travail exige donc de se risquer à tout instant malgré l'incertitude de s'engager devant l'indécidable" (Schwimmer, 2014 : 235). C'est bien à un tel travail de traduction pétri de nécessités de choisir des voies d'appropriation de la pratique dans la nébuleuse des offres de significations, que le genre réflexif académique confronte les futurs professionnels.

Dès lors, ce genre est appelé à naviguer entre informel et formel. Il peut parfois s'apparenter à un "petit genre de parole" (Volochinov, 2010), ouvert, soumis à l'oral à tous les aléas de la conversation ordinaire et du sens commun : c'est le cas notamment lorsque le tuteur de terrain et l'enseignant en formation discutent, le temps d'une pause informelle, des élèves qui semblent inattentifs, du matériel à préparer, de la prochaine séance avec les parents. Et du côté de l'écrit, ce sont des notes prises en cours d'action, 
des pensums, et autres formes d'écriture peu formelle outillant le "dialogue avec soimême". Il peut se structurer davantage, notamment dans le cadre des entretiens tripartites de bilan du stage entre $\mathrm{EF}, \mathrm{T}$ et $\mathrm{S}$, évoqués plus haut. Et à l'écrit, ce sont, outre des écritures intermédiaires en quête d'ordre et de structuration progressive, tous ces écrits professionnalisants que nous avons en partie énumérés. Au niveau formel le plus sophistiqué, outre les écrits de type rapport de stage, voire les travaux de recherche fondés sur des observations et interventions dans le milieu de travail, il convient de se pencher sur la fonction des récits écrits que les dispositifs sollicitent fréquemment, notamment des récits longs de formation dans lesquels les EF mettent en perspective les apprentissages professionnels cumulés au fil du temps.

Ce type de texte, le récit de formation pensé dans le cadre plus large du genre réflexif, réunit plusieurs exigences. Il renvoie notamment à la mobilisation de genres textuels mixtes. Avec Cifali (2007), on pourrait évoquer à cet égard, toutes proportions gardées, des dimensions génériques de l'essai, puisque le récit permet un ancrage et une illustration de savoirs professionnels acquis dans des situations et explicités à l'aide d'une démarche de connaissance scientifique ; de l'épopée, puisque des situations sont organisées comme des espaces-temps de l'émergence de ces apprentissages sur la base d'événements et d'enchainements multiples, de nouements et de dénouements, de rebondissements, à travers lesquels l'expérience s'éprouve entre agir, subir et pâtir ; et de l'autobiographie, puisque c'est à l'auteur que ces événements sont en principe arrivés.

\section{Un socle de caractéristiques communes dans le continuum oral- écrit}

37 Le genre réflexif académique, dans ses formats oraux ou écrits, confère aux textes dans lesquels il s'actualise, des caractéristiques compositionnelles proches: une langue dans un registre « intermédiaire » mixant les lexiques ordinaire et savant (lexique du discours professionnel), les segments de types de discours combinant narration et exposition, implication dans la situation ou au contraire distanciation, une certaine structure de présentation des problématiques professionnelles énoncées, des formes d'adressage vers les formateurs-évaluateurs, une énonciation qui se donne à la fois comme parole réflexive singulière et comme position dans un devenir professionnel, mais aussi une énonciation qui compose peu ou prou avec l'hétéronomie qui imprègne les systèmes de référence dans lesquels elle se fraie une cohérence.

38 Sur ces bases, en suivant Bronckart (1996), la sémiotisation de l'agir peut s'organiser de quatre manières différentes : 1 ) soit, sur un ancrage temporel dans la situation d'action proprement dite (monde discursif de l'exposer), soit, sur la distanciation temporelle par rapport à cette situation d'action (monde discursif du raconter) ; 2) l'agent, tel qu'il se présente dans le contenu sémiotisé, est impliqué (son rôle dans la situation d'action est sémiotisé) ou non (le discours se présente alors comme autonome). Quatre combinaisons sont possibles, donnant lieu à quatre types de discours : l'exposer impliqué, ou discours interactif ; l'exposer autonome, ou discours théorique ; le raconter impliqué, ou récit interactif et le raconter autonome, ou narration (Id.).

39 Les présentations de problématiques écrites dans les entretiens, les récits courts (microrécits de situations rencontrées en stage) ou longs (récit de la trajectoire de développement professionnel) montrent ainsi toutes sortes de combinaisons, comme dans cet extrait (repris dans Vanhulle, Pelladan-Dieci \& Balslev, 2015) : 
La motivation est le fruit des interactions qui se nouent entre le vouloir de l'élève, son pouvoir et le support social (...) [Exposé théorique autonome]. J'ai suggéré aux élèves après quelques cours où nos relations se détérioraient de faire le point sur ce qu'ils me reprochaient. (...). A mon grand étonnement, ils n'ont pas hésité à s'exprimer [exposé impliqué]. Il s'est avéré, entre autres, que la méthode déductive que je leur proposais leur posait beaucoup de difficultés d'adaptation et qu'ils préféraient une méthode inductive [Discours théorique conjoint à la situation de formation: les références aux méthodes renvoient au cours donné par le formateur qui lit ce texte]. Je me suis alors davantage orientée vers ce type de pédagogie et ils ont été très étonnés mais ravis que je tienne compte de leur remarque [récit interactif: adressage au formateur qui a explicitement demandé aux étudiants d'expliquer comment ils ont résolu des problèmes dans des situations de stages] (...). (Laure, enseignante en formation pour le degré secondaire, extrait portfolio). pour le traitement d'abord informatisé suivi ensuite d'une analyse approfondie de type herméneutique des textes réflexifs : systèmes de référence ; traitement (énoncés et modes énonciatifs) des significations liées à l'activité professionnelle en termes de savoirs, valeurs, normes, intentions ou motifs attribués à l'agir dans la construction de savoirs professionnels ; dynamiques interactionnelles et sociales ; empans techniques, contextuels, critiques et autoréférencés de la réflexivité ; régulations des conceptions, actions et sous-jacents.

\section{Systèmes de références} qui cadre le stage : inventaires de savoirs, compétences ou gestes professionnels attendus, orientations officielles, normes spécifiques de l'école de stage, guides pour les entretiens et l'évaluation. D'autres ancrent le discours dans les situations vécues dans le cours de l'agir avec les élèves, les tuteurs, les parents, avec les moments qui ont mis l'enseignant en formation en tension. D'autres encore proviennent du patrimoine de connaissances et d'expériences de vie. Les évocations de ces systèmes référentiels permettent à 
l'énonciateur de situer son discours, d'installer le contexte de son texte, et dans les interactions orales, de négocier des cadres qui contextualisent les contenus des échanges.

\section{Savoirs professionnels : énoncés et modes énonciatifs}

Les savoirs professionnels émergent de l'articulation, dans leur énoncé, de savoirs de référence transmis à l'université en tant que savoirs scientifiques (mais aussi orientés vers la prescription) ou issus de pratiques, conseils ou prescriptions transmises par les tuteurs de terrain et autres acteurs scolaires, d'une part, et de connaissances forgées dans les expériences vécues dans le cours de l'agir et donnant lieu à des formalisations, d'autre part. Leur ancrage dans des situations jugées emblématiques, événements problématiques, préoccupations pratiques mais aussi existentielles des EF laissent dans leur énonciation des marquages spatiotemporels, circonstanciels, interactionnels. Leur élaboration discursive entraine des opérations réflexives: réminiscence, décision, explication, attribution causale, interprétation, analyse, jugement, délibération. Ils se caractérisent également par des positionnements en termes de justification ou légitimation des actions évoquées en lien avec certaines intentions, motifs (en vue de ou parce que), de buts ou de valeurs.

Pour préciser les processus sémiotiques de traitement des contenus de savoirs professionnels et des prises de position qui les accompagnent, nous nous attachons aux indices textuels et sémiotiques rapidement inventoriés ci-après. La présence de ces indices dans les discours, et tout particulièrement les segments les plus saturés où ces indices se croisent, nous renseignent sur la construction du sens et ses méandres, sur les moments où un concept, un savoir émerge ou, le plus souvent, sur les tensions ou obstacles auxquels les EF sont confrontés dans la production de textes réflexifs débouchant sur la constitution de savoirs professionnels, surtout dans les textes écrits qui ne bénéficient d'aucun étayage oral.

C'est donc la combinatoire d'éléments issus des différentes strates textuelles et sémiotiques que l'analyse des discours tend à identifier, en l'occurrence sur la base de l'hypothèse suivante : les savoirs professionnels sont des systèmes conceptuels praxéologiques qui subsument aussi bien le caractère expérientiel des préoccupations sur lesquelles ils se sont élaborés, que le caractère strictement abstrait des concepts qu'ils intègrent. A la croisée de ces deux axes, ils sont nécessairement singuliers tout en s'accordant aux conditions sociales de leur production dans le dispositif de formation: cours et stages, contraintes du genre réflexif et des types de textes dans lesquels le genre doit s'actualiser (rapport de stage, journal de bord, récit de formation, analyse d'etc.).

Voici les catégories indicielles principalement privilégiées dans l'analyse. Au niveau de la textualisation, la construction des savoirs dépend notamment de l'usage des types de discours, entre exposé et narration (cf. Bronckart cité plus haut) comme modes de sémiotisation par le sujet de sa lecture des situations, de sa position en termes de rapports impliqués ou distanciés à ces situations, et de ses modalités d'adressage vers ses destinataires (formateurs). Les réseaux thématiques au niveau des contenus abordés, la structuration du raisonnement et de l'argumentation, mais aussi les filons isotopiques reliés au sens obvie ou latent (Tosi dans Tosi, Pellanda \& Vanhulle, 2014) que ces contenus acquièrent dans la mise en mots nous intéressent aussi, ce qui passe par une attention portée aux figures rhétoriques telles que métaphores, catachrèses, ellipses, etc. 

processus de sémiotisation du savoir, à travers notamment :

- Les modalisations discursives, que nous repérons selon les propositions interactionnistes sociodiscursives de Bronckart (1996) et des liens qu'il établit avec les réflexions d'Habermas (1987) à propos des mondes représentés : déontiques (ordre du devoir dans un monde de normes et de valeurs légitimées) ; appréciatives (ordre de l'agir et du pouvoir agir en tant qu'acteur dans le contexte concerné ou dans la profession en général) ; pragmatiques (ordre du faire, de l'efficace, de l'objectif visé) ; logiques (ordre de la loi, de la doxa, du devoir dans ses composantes juridiques ou symboliques) ; auxquelles nous ajoutons des modalisations libres (ordre de l'esthétique, de l'éthos, de la possibilité créatrice, du rapport au corps) ;

- Les dimensions mono- et polygérées du discours dans la prise en charge énonciative et l'imputation d'un point de vue (Rabatel, 2009 - cf. supra). En reformulant, restituant, réfutant, ou réélaborant un énoncé, l'énonciateur prend en charge un point de vue porté par l'énoncé et repris comme vrai, exact, discutable, pertinent.

- Le système des marqueurs de personnes et de circonstances spatiotemporelles: dans le système de prise en charge énonciative, le "on "ou le "nous "ou le "ils" peuvent renforcer selon les cas, la présence de l'énonciateur dans son propos, par le point de vue qui s'y expose: notamment parce que l'imputation du PDV (à des auteurs de référence, des formateurs, une communauté) sert d'argumentaire (Rabatel, 2009).

Allons plus avant dans le repérage des usages des déictiques. Ceux-ci contribuent à installer les situations évoquées comme sources de savoirs professionnels ou d'apprentissage, en particulier les situations relevées par les EF comme significatives, questionnantes ou problématiques. Ce n'est plus d'un point de vue "à propos de" qu'on parle ici, mais de la perspective donnée aux situations décrites : rapports de force ou de connivence entre "soi", "je", l'EF et "eux", les élèves, les parents, les collègues ; éclairages ou ombres portés sur ce qu'il se passe ici et maintenant, ou s'est passé ailleurs à un certain moment. Dans cette configuration des situations narrées ou exposées entrent également les différents connecteurs tels que "alors", "pourtant", "mais", etc.

A moins, comme cela se produit dans certains textes, qu'il n'en n'apparaisse aucun: la situation est alors lisse, comme si le temps était suspendu, l'espace flou et les événements non identifiables. L'absence flagrante de certains indices est elle-même une information pour l'analyse des discours : les savoirs professionnels émergent, ou pas, de l'analyse de situations, et de l'analyse des intentions que l'énonciateur veut imprimer à la compréhension de ses actions, sous "une certaine description" comme dirait Anscombe (1957). La question de l'évitement ou de l'incapacité actuelle à proposer une perspective descriptive et compréhensive se pose alors en termes d'apprentissage et de développement professionnel ou de difficulté à s'engager dans le dispositif proposé ou à en comprendre les buts.

\section{Dynamiques interactionnelles et sociales}

51 Cette catégorie ajoute aux critères précédents des modalités plus spécifiques de construction des savoirs dans les interactions orales (entretiens), en s'attachant aux opportunités que les interactants saisissent ou non pour formaliser des savoirs professionnels, à travers l'établissement de zones de compréhension, et compte tenu de rapports de places (dis-)symétriques, types d'interventions verbales (reformuler, ordonner, questionner, approuver, relancer...) et modes de participation à la progression 
thématique (Grize, 1996) : rétroactions, proactions ; effets de cohérence (filtrage, saillance, répétition). Pour les textes écrits, des éléments de cette catégorie peuvent être utiles pour identifier des effets de dialogisme dans les contenus et les prises en charge de points de vue.

\section{Empans réflexifs}

Avec Buysse (2009), on distingue quatre empans : techniques : prise en compte des moyens à mettre en œuvre par rapport aux savoirs à transmettre ou aux dimensions instrumentales de la gestion de la classe et des apprentissages. Contextuel : prise en compte des éléments pouvant amener à une compréhension des actions et des expériences éducationnelles, notamment en tenant compte du contexte de l'enseignement. Critique : prise en compte des enjeux sociopolitiques, éthiques, moraux, délibération sur des valeurs, des normes, des rapports entre des fins souhaitables et des moyens. Autoréférencé: l'énonciateur n'hésite pas à prendre ses propres références et expériences comme sources de réflexion.

\section{Régulations}

Cette catégorie travaillée par Buysse (2009) concerne les traces discursives indiquant des transformations que l'EF a opérées au niveau de ces conceptions, de ses actions ou des sous-jacents d'ordre affectif, motivationnel, intentionnel, qui facilitent ou qui contraignent son agir et son sentiment d'efficacité ou de bien-être.

\section{Une herméneutique des discours de formation}

Comme dit plus haut, c'est le croisement des indices et leur saturation dans des passages significatifs des textes, ainsi que le tissage plus implicite de significations au fil du texte dans des réseaux isotopiques, qui sont au centre des analyses des discours réflexifs. C'est là que la démarche contient une dimension herméneutique au-delà du repérage des indices. A défaut d'espace pour présenter précisément des analyses empiriques et des résultats, nous donnons ci-après une illustration de cette dimension.

Dans le registre des sens latents des discours, Tosi (notamment dans Tosi, Pellanda- Dieci \& Vanhulle, 2014) s'intéresse aux ambiguïtés, catachrèses, jeux des pronoms et des temps verbaux, pour saisir le travail du langage dans le développement professionnel des enseignants en formation. Dans l'exemple que nous lui empruntons ci-après ${ }^{6}$, il a mis en lumière à travers une analyse de discours approfondie toute la sémantique de la mise en danger de l'autorité professorale et du conflit autour du rapport ordinaire qui se joue normalement entre professeur et élèves.

Dans la situation présentée, un élève interpelle la professeure de français, passant de l'agacement à la colère, puis à l'accusation, pour finir par proférer des accusations dans un climat de plus en plus tendu, devant une classe tétanisée - tout un vocabulaire de la profanation ${ }^{7}$ et des passages par le passé simple servent à nourrir l'évocation du drame : une prof vit un événement menaçant tandis qu'elle s'efforce de raisonner l'élève et de se contenir, ce qui renvoie au thème de l'autorité et du self contrôle que l'enseignant est censé acquérir, selon les normes qui cadrent la formation et le travail de celui-ci : 
[...] nous étions en train de corriger des exercices [...] chaque élève lisait à tour de rôle sa réponse ; ensuite on procédait à la correction. [...] X lit sa phrase. Sa réponse [...] étant fausse, je le regarde et le lui dis [...] quelques autres élèves ont dit la bonne réponse [...]. Au moment où $\mathrm{X}$ entend cette réponse [...] il se lève sans mot dire et arpente littéralement la classe pour venir vers mon bureau [...] en me disant d'un ton agacé: "C'est ce que j'avais mis". Puis reprend sa feuille et repart s'asseoir.

[...] je lui dis en contenant mon calme que c'était dommage qu'il ait prononcé faux le pronom et qu'il n'avait pas besoin de se lever pour me montrer sa réponse correcte [...]. Je n'eus même pas le temps d'achever ma phrase que X commença tout à coup à se fâcher [...]. Il m'accusait [...] de lui réserver [...] un dur traitement accompagnés de reproches injustifiés. Cela dura quelques minutes, pendant lesquels la classe était silencieuse, presque tétanisée. Mes remarques pour ramener l'ordre ne suffirent pas, les tours étaient entrain de monter trop rapidement surtout parce que $\mathrm{X}$ proférait des accusations diverses et incompréhensibles. dans ce texte comme un topic, ou matrice de sens, enraciné dans la représentation du travail enseignant. Cette autorité, critère de reconnaissance de la compétence du professeur, est mise à mal par l'agression verbale et presque physique d'un élève, dans un contexte qui fonctionnait au départ selon des rituels bien établis: la correction d'exercices, la lecture chacun à son tour, la cohésion des élèves étonnés par l'attitude de leur pair et se rangeant du côté de la prof, médusés, tels un chœur de tragédie antique. La mise en texte théâtralise l'incident, à la mesure du séisme ressenti. La réflexivité consiste ici à questionner la pratique en termes d'amélioration d'une attitude ferme étant donné les caractéristiques des élèves (empan contextuel) ; elle ne s'engage pas pour autant dans une voie plus critique qui concernerait par exemple, les rapports d'autorité tels qu'ils sont orchestrés dans une forme scolaire ritualisée.

Cet exemple nous renvoie à la fois à deux constats fréquents de nos analyses. D'abord, non seulement l'écriture comme l'oralité réflexives ne coulent pas de source, et le passage à la régulation de l'agir ne découle pas non plus ipso facto de la réflexivité. Ensuite, la réflexivité prend appui sur ce qui fait souci à l'EF, elle peut alors s'y enliser au moins momentanément, tant les affects qui l'accompagnent restent dominants, ou au contraire se déployer vers un nouveau souci orienté vers l'amélioration ou régulation d'attitudes personnelles (sous-jacents affectifs, représentations), de modes d'action, ou de concepts revus par un travail critique d'investigation.

\section{En guise de conclusion}

La production de textes réflexifs par les $\mathrm{EF}$, comme le processus réflexif lui-même, ne coule pas de source ; il n'est même pas dit que l'écriture, voire l'oralité, soient des voies royales et exclusives pour une régulation satisfaisante des pratiques ni même pour une meilleure compréhension de celles-ci : l'injonction de la mise en discours est de produire des discours et des discours sur les discours, elle peut friser la sémiose illimitée et close sur elle-même, sans engendrer des actes réfléchis. Il nous paraît dès lors essentiel d'articuler le genre réflexif académique à une sémiotisation des situations professionnelles vécues, cette sémiotisation forcément subjective étant tendue vers une objectivation avec autrui des fondements de l'activité enseignante dans ses régularités, sous des angles didactiques, psychosociaux, pédagogiques, etc. D'une part, la sémiotisation de l'expérience constitue une forme de reconnaissance de celle-ci comme source de connaissance au contact de la réalité tout en insistant sur le caractère 
structurant de sa mise en mots, sans laquelle l'expérience appartiendrait au seuls flux et reflux de la vie sans pouvoir en être extraire. D'autre part, l'objectivation de situations, en dépit d'un caractère artificiel et orienté par les représentations en vigueur ici et maintenant (cf. les images de l'autorité), permet au processus de mise en texte de déboucher tendanciellement vers des régulations à partir de ces questions : et puis, que faire et comment?

La subjectivation comme processus d'élaboration des savoirs et du soi professionnels à travers des démarches discursives réflexives, écrites en particulier, renvoie non pas à une finalité du type "soyez authentiques dans votre réflexion sur votre agir professionnel et trouvez votre propre style", qui serait paradoxale et ambigüe dans le contexte multiréférentiel de la formation - forcément générateur de tensions cognitives affectant les possibilités énonciatives que les EF peuvent s'autoriser. Elle renvoie aux possibilités que l'EF peut se créer pour se développer au sein même de ce contexte qui délimite les conditions d'énonciation dans lesquels ses savoirs et son soi enseignant peuvent se construire.

61 A ce contexte, donc, de rendre visibles les opportunités de développement qu'il suggère, notamment par la mise en cohérence des attentes discursives multiples que l'injonction réflexive génère. Dans ce sens, la reconnaissance du genre réflexif serait une "affordance" (English, 2012) : identifier ses caractéristiques, en systématiser l'usage mais dans le sens des ouvertures qu'il offre (afford). Un genre se déploie dans les échanges sociaux à l'intérieur des structures et des formes qui le constituent en répondant à des modes de traitement de contenus particuliers ; plus il donne à voir ses caractéristiques, plus il offre à ses usagers des opportunités de développement par leur participation aux échanges.

Une telle constitution d'un genre réflexif reconnu dans la sphère académique doit être pensée, au-delà de sa pratique disséminée dans les différentes disciplines en fonction de leurs objets spécifiques, dans une perspective transdisciplinaire : pas plus que le travail de l'enseignant, le genre réflexif n'a de frontières conceptuelles; d'académique il peut devenir un genre professionnel, faisant "feu de tout bois". Au final, ce sont bien les pratiques qui forment systématiquement les objets dont parlent les discours (Foucault, 1969 : 67).

\section{BIBLIOGRAPHIE}

ANScOMBE, E. (1957). Intention, Londres, Basil Blackwell.

BALSLEV, K. \& TOMINSKA, E. (2012). « Opportunités de construction de savoirs professionnels dans les entretiens de stage ». Travail et Apprentissages 9, p. 163-182. En ligne : https://archiveouverte.unige.ch/unige:23083.

BAlSlev, K., DobrowolsKa, D., PERRÉARD-Vité, A., VANHulle, S., TOMinSKA, E. \& MOSQUERA, S. (2014). « Voicing Experience in Order to Transform it in Professional Knowledge ». 4th International Conference on Applied Linguistics and Professional Practice (ALAPP), septembre, Université de Genève. 
BEAUDET, C. \& REY, V. (2012). « De l'écrit universitaire à l'écrit professionnel : comment favoriser le passage de l'écriture heuristique et scientifique à l'écriture professionnelle? ». Scripta 30, p. 169-193.

BLASER, C. \& POLLET, M.-C. (coords). (2010). L'appropriation des écrits universitaires. Namur : Presses universitaires de Namur.

вотА, С. (2008). « Eugenio Coseriu : linguistique et philosophie du langage, un modèle complexe du fonctionnement langagier ». Texto! XIII (1/2). En ligne : http://www.revue-texto.net/ index.php?id=102.

BRONCKART, J.-P. (1996). Activité langagière, textes et discours. Pour un interactionnisme sociodiscursif. Neuchâtel : Delachaux et Niestlé.

- (2005). Une introduction aux théories de l'action. Les carnets de la section des sciences de l'éducation. Genève : Faculté de psychologie et des sciences de l'éducation.

BRONCKART, J.-P. \& BOTA, C. (2011). Bakhtine démasqué. Histoire d'un menteur, d'une escroquerie et d'un délire collectif. Genève : Droz.

BULEA, E. (2011). «Compétence langagière et compétence professionnelle : éléments pour une approche intégrée ». Bulletin VALS-ASLA 93, p. 69-84.

BRANCA-ROSOFF, S. (1999). « Types, modes et genres : entre langue et discours ». Langages et Sociétés 87, p. $5-24$.

BUYSSE, A. (2009). « Médiations contrôlantes et structurantes : une base pour penser la formation ». Revue suisse des sciences de l'éducation 31 (3), p. 585-602.

CIFALI, M. \&ANDRÉ, A. (2007). Écrire l'expérience. Paris : Presses universitaires de France.

COSERIU, E. (2001) [1968]. L'homme et son langage. Louvain/Paris : Peeters.

DAUNAY, B. (2011). Les écrits professionnels des enseignants : approche didactique. Rennes : Presses universitaires de Rennes.

DELCAMBRe, I., \& LAHANIER-REUTER, D. (2012). «Difficultés de l'écriture académique en Sciences Humaines et perceptions de l'accompagnement : analyse de discours d'étudiants». Diptyque, p. 37-61.

DELORMAS, P. (2012). « Contribution à une analyse de l'ethos discursif de l'enseignant en formation, envisagé à travers le critère de la réflexivité ». Pratiques 153-154, juin, p. 100-114.

DEWEY, J. (1993). Logique. La théorie de l'enquête, Paris, Presses universitaires de France.

- (2011). La formation des valeurs. Trad. de l'anglais par A. Bidet, L. Quéré \& G. Truc. Paris, La Découverte.

DOBRowolsKA, D. (2014). «Ethos discursif dans les problématiques professionnelles chez les enseignants en formation ». $4^{\text {th }}$ International Conference on Applied Linguistics and Professional Practice (ALAPP), septembre, Université de Genève..

DUFAYS, J.-L. \& THYRION, F. (2004). Réflexivité et écriture en formation des enseignants. Louvain-La Neuve : Presses universitaires de Louvain.

ENGLISH, F. (2012). « Écrire différemment : repenser le genre ». Pratiques 153-154, juin, p. 177-194.

GIDDENS, A. (2005). La constitution de la société. Paris : Presses universitaires de France.

GRIZE, J.-B. (1996). Logique naturelle et communications. Paris : Presses universitaires de France.

FOUCAULT, M. (1969). L'archéologie du savoir. Paris : Gallimard. 
HOFSTETTER, R. \& SCHNEUWLY, B. (éds) (2007). Savoirs en formations. Bruxelles : De Boeck. JORRO, A. (2011). « Éthos professionnel et transactions de reconnaissance ». In: Jorro, A.\& De Ketele, J.-M. (éds), La professionnalité émergente : quelle reconnaissance? Bruxelles : De Boeck, p. 51-64.

HABERMAS, J. (1987). Théorie de l'agir communicationnel, I et II. Paris : Fayard.

LEBLAY, C. (2014). « Les écritures intermédiaires réflexives en littératie avancée ». Le Français aujourd'hui, 184, p. 103-115.

MARCEL, J.-F. (éd.) (2010). « Le développement professionnel : quels indicateurs ?». Questions vives 5 (11).

MAINGUENEAU, D. (2013). « L'èthos : un articulateur ». COnTEXTES 13. En ligne : http://

contextes.revues.org/5772.

MEZIROW, J. (2001). Penser son expérience. Lyon : Chronique sociale.

MOIRAND, S. (2000). « Variations discursives dans deux situations contrastées de la presse ordinaire ». Les Carnets du Cediscor 6, p. 45-62.

MORISSE, M. (2003). «Écriture et réflexivité, quel(s) rapport(s) ? ». Perspectives documentaires en éducation 58, p. 61-67.

- (2012). «La production de supports didactiques comme processus de réflexivité chez les enseignantes et enseignants ». In: Morisse, M., Lafortune, L. \& Cros, F., Se professionnaliser par l'écriture. Quels accompagnements? Québec: Presses universitaires du Québec, p. 153-180.

MOSQUERA, S. (2014). « Rencontres, ruptures et réélaborations des savoirs professionnels d'un enseignant débutant : analyse de leur transition entre la formation initiale et l'entrée dans le métier ». $4^{\text {th }}$ International Conference on Applied Linguistics and Professional Practice (ALAPP), septembre, Université de Genève.

PELLANDA-DIECI, S. \& TOSI, J.-M. (2014). « Des préoccupations des enseignants débutants aux différentes facettes du métier ». Diversité 177, p. 99-108.

PellandA-DieCI, S., BALSLEV, K., TOSI, M. \& VANHUlLE, S. (2012). « Indices de développement professionnel dans les écrits d'étudiants en formation initiale à l'enseignement secondaire ». Colloque international Stratégies d'écriture, stratégies d'apprentissage de la maternelle à l'Université, IUFM Alsace, Colmar, France, mars.

PERRENOUD, P. (2001). Développer la pratique réflexive dans le métier d'enseignant. Professionnalisation et raison pédagogique. Paris : ESF.

RABATEL, A. (2005). « La part de l'énonciateur dans la construction interactionnelle des points de vue ». Marges linguistiques 9, mai, p. 115-136.

- (2009). «Prise en charge et imputation, ou la prise en charge à responsabilité limitée ». Langue française 161, juin, p. 71-88.

RICARD-FERSING, E. \& CRINON, J. (2004). « Action, valeurs et normes professionnelles dans les écrits de formation ", Repères 30, p. 87-99.

SCHÖN, D. (1996). «À la recherche d'une nouvelle épistémologie de la pratique et de ce qu'elle implique pour l'éducation des adultes ». In: Barbier, J.-M. , Savoirs théoriques et savoirs d'action. Paris : Presses universitaires de France, p. 201-222.

SCHWIMMER, M. (2014). « Traduire la recherche en pratiques : vers un acte de transformation et d'engagement ». Traductions. Revue suisse des sciences de l'éducation 36 (2), p. 229-242. 
TOSI, J-M., PELLANDA-DIECI, S. \& VANHULLE, S. (2014). « Les récits de formation, entre sens obvie et sens latent ». Congrès International Writind Research Across Borders. Université de Paris Ouest Nanterre La Défense, 19-22 février.

- (2007). « Savoir professionnels et construction sociodiscursive de l'agir ». Bulletin VALS-ASLA 90 , p. $167-188$.

VANHUlle, S. (2009). Des savoirs en jeu au savoir en "je”. Cheminements réflexifs et subjectivation des savoirs chez de jeunes enseignants en formation. Berne : Peter Lang.

- (2011). « Comprendre le développement professionnel par l'analyse des discours ». Scripta 15 (28), p. $145-170$.

- (2015). «(Se) former dans l'alternance : des mondes de discours en dés-équilibre ». In: Balslev

K., Cartaut, S., Filliettaz, L. \& Vinatier, I., La part du langage : pratiques professionnelles en formation. Paris : L'Harmattan.

VAnHUlle, S. PellandA-DieCI, S. \& BALSLEV, K. (2015). « Indicators of Professional Development in Texts Written by Prospective Teachers ». Journal of Cognitive Education and Psychology 14 (1), p. 4-27.

VERMERSCH, P. (1994). L'entretien d'explicitation en formation initiale et en formation continue. Paris : ESF.

volochinov, V. (1981). « Le discours dans la vie et le discours dans la poésie ». In: Todorov, T. (éd.), Mikhail Bakhtine le principe dialogique. Paris : Seuil, p. 181-215.

- (2010). Le marxisme et la philosophie du langage. Limoges : Lambert-Lucas.

VYGOTSKI, L. S. (1997). Pensée et langage. Paris : La Dispute.

VYGoTSKI, L. (2003). Conscience, inconscient, émotion. Paris : La Dispute.

\section{NOTES}

1. Chez Bronckart (e.a. 2005), l'agir renvoie à toute forme d'intervention orientée d'un ou de plusieurs humains dans le monde, par exemple un travail structuré en tâches, et se décline dans le déroulement temporel d'un cours de l'agir. L'activité désigne une lecture de l'agir impliquant des dimensions motivationnelles et intentionnelles mobilisées au niveau collectif, et l'action une lecture de l'agir impliquant ces dimensions mobilisées par des agents.

2. Dans notre système de formation des enseignants du premier degré (classes d'élèves de 4 à 12 ans) en huit semestres, les 5 premiers semestres se fondent sur des "modules d'alternance " entre les cours à l'université et des périodes de pratiques et observations dans des classes ; ensuite viennent 18 semaines de "stages en responsabilité " qui sont réparties sur les trois derniers semestres. Les «stages en responsabilité » désignent des stages organisés dans le cursus même de la formation initiale et lors desquels les stagiaires sont accueillis par un enseignant en exercice qui leur confie sa classe tout en restant présent, participant de la sorte activement à la formation du stagiaire par l'observation de l'activité de celui-ci, la co-responsabilité ponctuelle et la délégation quasi-totale de la responsabilité de la tenue de la classe en sa présence quasipermanente. Pour les enseignants du second degré (classes d'élèves de 12 à 18 ans), les stagiaires sont totalement responsables de classes à mi-temps pendant un an, et des référents universitaires et de terrain externes les supervisent. Nos dispositifs prévoient la tenue de portfolios ou dossiers de développement professionnel (DDP) dans les deux degrés, primaire et secondaire, avec diverses nuances impossibles à détailler ici, mais s'intégrant de part et d'autre dans des artéfacts structurés par des interactions avec les pairs, l'évaluation conjointe avec les formateurs, et, pour le primaire, le partenariat avec les tuteurs du terrain dans des entretiens 
oraux tripartites s'appuyant en partie sur la présentation par l'étudiant d'une problématique préalablement écrite et versée au portfolio, suivie d'une discussion entre les trois acteurs qui élargit les échanges à l'ensemble du stage (enseignant en formation EF, superviseur universitaire $\mathrm{S}$ et tuteur terrain $\mathrm{T}$ ).

3. Recherche financée par le Fonds National Suisse de la Recherche ( $\left.{ }^{\circ} 137959\right)$ : La construction de savoirs professionnels dans les stages en enseignement primaire (2012-2015). Onze EF (soit, une trentaine d'entretiens impliquant des $\mathrm{T}$ et $\mathrm{S}$ différents qui suivent ces stagiaires sur une année de stages en responsabilité). Un entretien (allant de 900 à 2200 tours de parole en 1h30 environ) retranscrit compte de 20 à 40 pages format $\mathrm{A} 3$.

4. A ce stade, une trentaine de DDP analysés, produits par les étudiants en formation en enseignement secondaire de 2011 à 2012. Les micro-récits comptent à peine quelques lignes, chaque portfolio en contient au moins 6 ; le portfolio se clôture par un récit final de la trajectoire d'une quinzaine de pages.

5. Le lecteur intéressé peut se reporter à la page de Groupe de recherche TALES (Kristine Balslev, Cristian Bota, Alexandre Buysse, Dominika Dobrowolska, Santiago Mosquera, Sandra Pellanda Dieci, Anne Perréard Vité, Edyta Tominska, Jean-Marc Tosi, Dir. Sabine Vanhulle) : http:// www.unige.ch/fapse/tales.

6. Corpus de plus de quatre cents Dossiers de développement professionnel d'enseignants en formation (degré secondaire) réunissant des textes réflexifs, des récits essentiellement, constitué par Pellanda, Dieci et Tosi depuis 2011.

7. Tosi montre tout le rapport qui s'établit dans le réseau isotopique de cette partie complète du récit, plus étendue que notre extrait, autour des termes " proférer » puis " profaner »; il montre également comment les temps verbaux, en particulier ici le passé simple renvoie littéralement à une dramaturgie de l'événement narré, sur lequel l'auteure ne revient que très peu de manière métaréflexive. La situation est littéralement « revécue » dans une écriture soudain marquée par de nombreux lapsus et fautes orthographiques et lexicales, le reste du récit de dix pages environ s'avérant correct et de bonne tenue.

\section{RÉSUMÉS}

L'écriture réflexive possède une fonction structurante dès lors qu'elle engage le professionnel en formation dans une énonciation marquée par un projet d'enquête à propos de son agir. Elle l'appelle à identifier des situations de travail qui l'incitent à décider de ses actions et à en jauger les conséquences. Il lui faut trouver les mots pour saisir en quoi ces situations - éprouvées, subies ou agies - constituent des expériences d'apprentissage cruciales car porteuses de transformations du soi professionnel, des savoirs du métier, des possibilités propres d'agir. La dimension réflexive de l'écriture peut alors enclencher un processus de subjectivation du sens, au-delà de la conceptualisation objectivante qu'offre l'usage de savoirs scientifiques. L'agir s'incarne et peut se réguler dans cette subjectivation. Nous envisageons cet usage de l'écriture sous l'angle d'un "genre réflexif académique" qui tend à relier intimement les enseignants en formation à leur profession. Se déployant dans un continuum de divers types de textes oraux et écrits, ce genre s'étend depuis des "petits genres de parole" tels que des conversations informelles, vers la construction de textes très structurés tels que des récits de trajectoire de formation qui articulent des dimensions théoriques, narratives et autoréférencées dans une mise en perspective des fondements récurrents du travail enseignant. Ce genre très particulier à 
l'université présente ainsi des exigences littéraciques élevées. Nous en explorons les caractéristiques, compte tenu non seulement de composantes linguistiques et textuelles mais aussi des conditions sociales et interactionnelles dans lesquelles il se déploie, pour esquisser quelques pistes en vue d'outiller la production de textes réflexifs de haut niveau. Ces propositions se concrétisent dans un instrument d'analyse des discours d'apprentissage professionnel (ADAP), que nous avons élaboré au fil de nos travaux relatifs aux processus discursifs de la construction des savoirs et du soi professionnels chez les enseignants en formation.

INDEX

Mots-clés : genre réflexif, subjectivation, savoirs professionnels, situation expérienciée, régulation

\section{AUTEUR}

\section{SABINE VANHULLE}

Université de Genève, Groupe de recherche TALES/TALK, Suisse 